\title{
Selective embolization of ruptured feeding artery aneurysm followed by resection of arteriovenous malformation
}

\author{
Brian P. Walcott, MD, Jonathan J. Russin, MD, Robin Babadjouni, BS, and William J. Mack, MD \\ Department of Neurological Surgery, Keck School of Medicine, University of Southern California, Los Angeles, California
}

This is the case of a man in his 40 s who suffered sudden collapse into a deep coma as a result of a ruptured arteriovenous malformation (AVM) feeding artery aneurysm within the lateral ventricle. The ruptured aneurysm was successfully treated with Onyx embolization of the feeding pedicle. The AVM and the feeding artery aneurysm were then removed via a transcallosal approach. This case highlights the utility of interrogating the AVM with microcatheterization of the feeding pedicles in order to define the exact anatomical features necessary for treatment planning. It also reviews the anatomy of the choroidal fissure.

The video can be found here: https://youtu.be/UeqFzhTRU1Q.

KEYWORDS AVM; embolization; choroidal fissure; transcallosal; surgery 